\title{
Automatic Modulation Classification Using PDF Approximation
}

\author{
Yong Jin, Shuichi Ohno, and Shinichi Tokuhara \\ E-mail: \{jinyong2009,ohno\}@hiroshima-u.ac.jp, tshinichi0813@gmail.com
}

\begin{abstract}
In this paper, we consider automatic modulation classification (AMC) from received signals over flat fading channels. AMC is a technique to identify the modulation type of the transmitted symbols by using received symbols. First, we develop a method to determine the modulation type based on the Neyman-Pearson (NP) detector. Then, to reduce the numerical complexity, a method by using probability density function (PDF) approximation is derived. To certify our detector can be scarcely effected by the uncertainty in noise variance, we compare the case that the noise variance is available with the case that the noise variance is unavailable. Furthermore numerical simulations are conducted to assess the performance of our proposed methods even when only finite samples are limited.
\end{abstract}

\section{Introduction}

As the number of wireless devices gets larger, the shortage of frequency spectrum becomes more serious. Therefore, how to improve spectrum utilization efficiently has been realized as a challenging topic in wireless communication. Cognitive radio networks have been developed for the efficient use of the bandwidth. In cognitive radio networks, there are two categories of users; primary users and secondary users. Secondary users are allowed to transmit signals if primary users sharing the same band but do not transmit signals, i.e., are idle. Cognitive radio enables efficient use of the communication resources but requires the detection of the presence of the transmission of primary users, which is also called spectrum sensing $[1,2]$. With a good design of spectrum sensing algorithm, spectrum utilization could be efficiently improved.

Many detection schemes have been proposed. The detectors using the energy of the received signals are the most commonly applied since they do not require any prior knowledge about the structure of primary users' signals [3]. The detectors that exploit the statistics of the transmitted signals, including cyclostationarity [4] and higher-order statistics [5], have been also available. They have been originally developed to classify the modulation of the received signals, which are referred to as automatic modulation classification (AMC). However, relatively large number of received signals are required to capture the cyclostationarity and higher-order statistics.
The energy based detectors are derived from likelihood ratio test (LRT) [6]. If the true statistics of the received signals are available, then the LRT is asymptotically optimal as the number of received signals goes to infinity. The energy detector requires the knowledge of the noise variance during the detection. However, the noise variance could not be known in general and the uncertainty of the noise variance may significantly degrade the performance of the energy detector. The optimality of generalized likelihood ratio test (GLRT) with a finite number of received signals has been also established in [7]. The GLRT based spectral sensing which utilizes some prior information has been proposed in [8].

In this paper we consider automatic modulation classification (AMC) from received signals over flat fading channels. First we develop a method to determine the modulation type based on the Neyman-Pearson (NP) detector. Then, to reduce the numerical complexity, a method by using probability density function (PDF) approximation is derived. Since some detectors require the value of the noise variance (e.g. energy detector), we compare the case that the noise variance is available with the case that the noise variance is unavailable. Then we provide some simulation results to demonstrate the performance of our proposed methods even when we have a small number of samples.

\section{Problem Formulation}

Let $s_{k}$ be the $k$ th transmitted symbol. We assume that after some processing, the received signal can be expressed as

$$
y_{k}=h s_{k}+w_{k}, \quad k=1, \ldots, K
$$

where $K$ is the number of samples, $h$ is the channel coefficient. And $w_{k}$ is the Gaussian noise with 0 mean and variance $\sigma_{w}^{2}$.

Let us normalize the received symbol energy as

$$
E\left\{\left|h s_{k}\right|^{2}\right\}=\sigma_{s}^{2}=1 .
$$

For simplicity of the presentation, we assume that $\left\{s_{k}\right\}_{k=1, \ldots, K}$ are drawn from either Quadrature PSK (QPSK) constellation or 8 quadrature amplitude modulation (QAM) constellation, whose signal points are $\{ \pm 1, \pm j\}$ or $\{ \pm 1 \pm j, \pm(1+\sqrt{3}), \pm j(1+\sqrt{3})\}$. Then, - 360 our detection problem can be formulated as a classical 
detection problem such that

$$
\begin{gathered}
\mathcal{H}_{0}: s_{k} \text { in } y_{k}(k=1, \ldots, K) \text { are QPSK symbols } \\
\mathcal{H}_{1}: s_{k} \text { in } y_{k}(k=1, \ldots, K) \text { are 8QAM symbols }
\end{gathered}
$$

where $\mathcal{H}_{0}$ is the hypothesis that QPSK symbols are transmitted, while $\mathcal{H}_{1}$ is the hypothesis that $8 \mathrm{QAM}$ symbols are transmitted.

We denote the probability density function (PDF) of $y_{k}$ under $\mathcal{H}_{i}$ as $p\left(y_{k} \mid \mathcal{H}_{i}\right)$. The false alarm is the probability that $\mathcal{H}_{1}$ is mistakenly selected, which we denote $P_{F A}:=P\left(\mathcal{H}_{1} \mid \mathcal{H}_{0}\right)$, where $P(A \mid B)$ is the probability of an event $A$ conditioned on an event $B$. On the other hand, $P_{D}:=P\left(\mathcal{H}_{i} \mid \mathcal{H}_{i}\right)$ is the probability of (correct) detection. The probability $P_{m}$ of miss detection is given by $P_{m}=1-P_{D}$.

If $p\left(y_{k} \mid \mathcal{H}_{i}\right)$ are available, then the optimal detector that maximizes the probability $P_{D}$ of correct detection for a given $P_{F A}=\alpha$ is the Neyman-Pearson (NP) detector, which decides $\mathcal{H}_{1}$ if

$$
L(\boldsymbol{y})=\frac{p\left(\boldsymbol{y} \mid \mathcal{H}_{1}\right)}{p\left(\boldsymbol{y} \mid \mathcal{H}_{0}\right)}>\gamma
$$

where $\boldsymbol{y}=\left[y_{1}, \ldots, y_{K}\right], p\left(\boldsymbol{y} \mid \mathcal{H}_{i}\right)=\Pi_{k=1}^{K} p\left(y_{k} \mid \mathcal{H}_{i}\right)$ is the PDF of $\boldsymbol{y}$ conditioned on $\mathcal{H}_{i}$, and $\gamma$ is the threshold determined by

$$
P_{F A}=\int_{\boldsymbol{y}: L(\boldsymbol{y})>\gamma} p\left(\boldsymbol{y} \mid \mathcal{H}_{0}\right) d y=\alpha .
$$

\section{Proposed methods}

Without the knowledge of the channel coefficients, it is not possible to estimate the transmitted symbols from the received signals. Thus, in place of $y_{k}$, we will utilize $r_{k}=\left|y_{k}\right|$ for our automatic modulation classification (AMC). $p\left(\boldsymbol{r} \mid \mathcal{H}_{0}\right)$ is the PDF of $\boldsymbol{r}$ under the hypothesis $\mathcal{H}_{0}$, while the PDF of $\boldsymbol{r}$ under the hypothesis $\mathcal{H}_{1}$ is $p\left(\boldsymbol{r} \mid \mathcal{H}_{1}\right)$.

Under the hypothesis $H_{0}$, when QPSK symbols are transmitted, whose constellation points are $\{ \pm 1, \pm j\}$. The first term $h s_{k}$ on the R.H.S. of (1) has zero mean and variance $\sigma_{s}^{2}$. Also the condition $\sigma_{s}^{2}=1$ in (2) is satisfied. Since the PDF of $r_{k}$ is Rician distributed, it can be expressed as

$$
p\left(r_{k} \mid \mathcal{H}_{0}\right)=\frac{2 r_{k}}{\sigma_{w}^{2}} \exp \left(-\frac{r_{k}^{2}+\sigma_{s}^{2}}{\sigma_{w}^{2}}\right) I_{0}\left(2 \frac{r_{k} \sigma_{s}}{\sigma_{w}^{2}}\right)
$$

where

$$
I_{0}(\eta)=\frac{1}{\pi} \int_{0}^{\pi} e^{\eta \cos \phi} d \phi
$$

is the modified Bessel function of the first kind and zeroth order.

Meanwhile, under the hypothesis $\mathcal{H}_{1}$, only 8QAM symbols are transmitted. Since the energy of 8QAM symbols is not 1 , we need to normalize it. From the constellation points of 8QAM signals, which are
$\{ \pm 1 \pm j, \pm(1+\sqrt{3}), \pm j(1+\sqrt{3})\}$, the variance can be determined by

$$
\frac{1}{2}\left[(1+1)+(\sqrt{3}+1)^{2}\right]=3+\sqrt{3} .
$$

Since the variance need to be normalized as 1 , from (9) we should multiply $d=\sqrt{\frac{1}{3+\sqrt{3}}}$ to each 8QAM symbol.

Furthermore, the inner side of 8QAM signals have zero mean and variance $\sigma_{s_{1}}^{2}=2 d^{2}$, while the outer side of 8QAM signals have zero mean with variance $\sigma_{s_{2}}^{2}=$ $(4+2 \sqrt{3}) d^{2}$.

In the case of $\mathcal{H}_{1}$, the PDF of $r_{k}$ is also Rician distributed, which is expressed as

$$
\begin{aligned}
p\left(r_{k} \mid \mathcal{H}_{1}\right) & =\frac{1}{2}\left\{\frac{2 r_{k}}{\sigma_{w}^{2}} \exp \left(-\frac{r_{k}^{2}+\sigma_{s_{1}}^{2}}{\sigma_{w}^{2}}\right) I_{0}\left(2 \frac{r_{k} \sigma_{s_{1}}}{\sigma_{w}^{2}}\right)\right. \\
& \left.+\frac{2 r_{k}}{\sigma_{w}^{2}} \exp \left(-\frac{r_{k}^{2}+\sigma_{s_{2}}^{2}}{\sigma_{w}^{2}}\right) I_{0}\left(2 \frac{r_{k} \sigma_{s_{2}}}{\sigma_{w}^{2}}\right)\right\}
\end{aligned}
$$

We assume that the transmitted symbols $s_{1}, \ldots, s_{K}$ are independent of each other. Then, we obtain

$$
p\left(\boldsymbol{r} \mid \mathcal{H}_{i}\right)=\Pi_{k=1}^{K} p\left(r_{k} \mid \mathcal{H}_{i}\right) .
$$

From (11), one can compute the likelihood ratio of $\boldsymbol{r}=$ $\left[r_{1}, \ldots, r_{K}\right]$ with the PDF of $r_{k}$ under $\mathcal{H}_{0}$ and $\mathcal{H}_{1}$ to obtain the optimum detector as follows:

$$
\begin{aligned}
L(\boldsymbol{r}) & =\frac{p\left(\boldsymbol{r} \mid \mathcal{H}_{1}\right)}{p\left(\boldsymbol{r} \mid \mathcal{H}_{0}\right)} \\
& =\Pi_{k=1}^{K} \frac{\frac{1}{2}\left[e^{-\sigma_{s_{1}}^{2}} I_{0}\left(2 \frac{r_{k} \sigma_{s_{1}}}{\sigma_{w}^{2}}\right)+e^{-\sigma_{s_{2}}^{2}} I_{0}\left(2 \frac{r_{k} \sigma_{s_{2}}}{\sigma_{w}^{2}}\right)\right]}{e^{-\sigma_{s}^{2}} I_{0}\left(2 \frac{r_{k} \sigma_{s}}{\sigma_{w}^{2}}\right)} \\
& \lessgtr_{\mathcal{H}_{1}}^{\mathcal{H}_{0}} \gamma .
\end{aligned}
$$

When the condition $L(\boldsymbol{r})>\gamma$ is satisfied, the hypothesis $\mathcal{H}_{1}$ is determined, which means in this case 8QAM symbols are transmitted at the transmitter side. On the other hand, when $L(\boldsymbol{r})<\gamma$ is met, the hypothesis $\mathcal{H}_{0}$ is determined, which means only QPSK symbols are transmitted. For the threshold $\gamma$, it is determined by the false alarm as in (6). However, the optimal detector in (12) requires a large amount of computations.

To develop the detector with less computations, we approximate the PDF of $r_{k}$ as Gaussian. Under the case of the hypothesis $\mathcal{H}_{0}$, the PDF of $r_{k}$ is expressed as

$$
p\left(r_{k} \mid \mathcal{H}_{0}\right)=\frac{1}{\sqrt{2 \pi \sigma_{w}^{2}}} \exp \left(-\frac{\left(r_{k}-\sigma_{s}^{2}\right)^{2}}{\sigma_{w}^{2} / 2}\right) .
$$

Meanwhile, under the case of the hypothesis $\mathcal{H}_{1}$, the PDF of $r_{k}$ can be expressed as

$$
\begin{aligned}
p\left(r_{k} \mid \mathcal{H}_{1}\right) & =\frac{1}{2}\left\{\frac{1}{\sqrt{2 \pi \sigma_{w}^{2}}} \exp \left(-\frac{\left(r_{k}-\sigma_{s_{1}}^{2}\right)^{2}}{\sigma_{w}^{2} / 2}\right)\right. \\
& \left.+\frac{1}{\sqrt{2 \pi \sigma_{w}^{2}}} \exp \left(-\frac{\left(r_{k}-\sigma_{s_{2}}^{2}\right)^{2}}{\sigma_{w}^{2} / 2}\right)\right\} .
\end{aligned}
$$


So that the likelihood ratio is obtained as

$$
L(\boldsymbol{r})=\Pi_{k=1}^{K} \frac{\frac{1}{2}\left[e^{-\left(r_{k}-\sigma_{s_{1}}^{2}\right)^{2}}+e^{-\left(r_{k}-\sigma_{s_{2}}^{2}\right)^{2}}\right]}{e^{-\left(r_{k}-\sigma_{s}^{2}\right)^{2}}} .
$$

Taking the logarithm of (15) and using the approximation of the log-sum-exp function such that

$$
\log \left(e^{x_{1}}+e^{x_{2}}+\cdots+e^{x_{N}}\right) \approx \max \left(x_{1}, x_{2}, \ldots, x_{N}\right)
$$

then we reach to

$$
\begin{aligned}
& \log L(\boldsymbol{r}) \approx-\sum_{k=1}^{K} \min \left\{\left(r_{k}-\sigma_{s_{1}}^{2}\right)^{2},\left(r_{k}-\sigma_{s_{2}}^{2}\right)^{2}\right\} \\
&+\sum_{k=1}^{K}\left(r_{k}-\sigma_{s}^{2}\right)^{2}-K \log 2 . \\
& \lessgtr_{\mathcal{H}_{0}}^{\mathcal{H}_{1}} \gamma
\end{aligned}
$$

which is utilized as our detector.

If $L(\boldsymbol{r})>\gamma$ is satisfied, the hypothesis $\mathcal{H}_{1}$ is determined, which means in this case 8QAM symbols are transmitted at the transmitter side. But if $L(\boldsymbol{r})<\gamma$ is met, the hypothesis $\mathcal{H}_{0}$ is determined, which means only QPSK symbols are transmitted. And the threshold $\gamma$ is also determined by the false alarm as in (6). Compare the detection function (17) to (12), computational complexity is visibly reduced.

From (1) we can derive the energy of received signals as

$$
E\left\{\left|y_{k}\right|^{2}\right\}=\sigma_{s}^{2}+\sigma_{w}^{2} .
$$

If $\sigma_{w}^{2}$ is unknown but sufficient small compared to $\sigma_{s}^{2}$, normally a natural choice for its estimate is

$$
\hat{\sigma}_{s}^{2}=\frac{1}{K} \sum_{k=1}^{K}\left|y_{k}\right|^{2}
$$

For numerical simulation, it is available to use $\hat{\sigma}_{s}^{2}$ in place of true $\sigma_{s}^{2}$.

But if $\sigma_{w}^{2}$ is known, then the estimate should be

$$
\hat{\sigma}_{s}^{2}=\frac{1}{K} \sum_{k=1}^{K}\left|y_{k}\right|^{2}-\sigma_{w}^{2}
$$

In this paper, note that theoretical likelihood ratio in (12) requires the values of both $\sigma_{s}^{2}$ and $\sigma_{w}^{2}$. While the approximated likelihood ratio in (17), it is not necessary to know $\sigma_{w}^{2}$.

\section{Simulation Results}

We define signal energy as $E\left\{\left|s_{k}\right|^{2}\right\}=\mathcal{E}_{s}$ (where $E\{\cdot\}$ is called the expected value operator) and set the noise variance to be $\sigma_{w}^{2}=\mathcal{N}_{0}$, and define signal to noise ratio $(\mathrm{SNR})$ as $10 \log \left(\mathcal{E}_{s} / \mathcal{N}_{0}\right)$ in $\mathrm{dB}$.

To demonstrate the performance of our proposed methods, we set the number of samples $K$ as $K=1,50$.

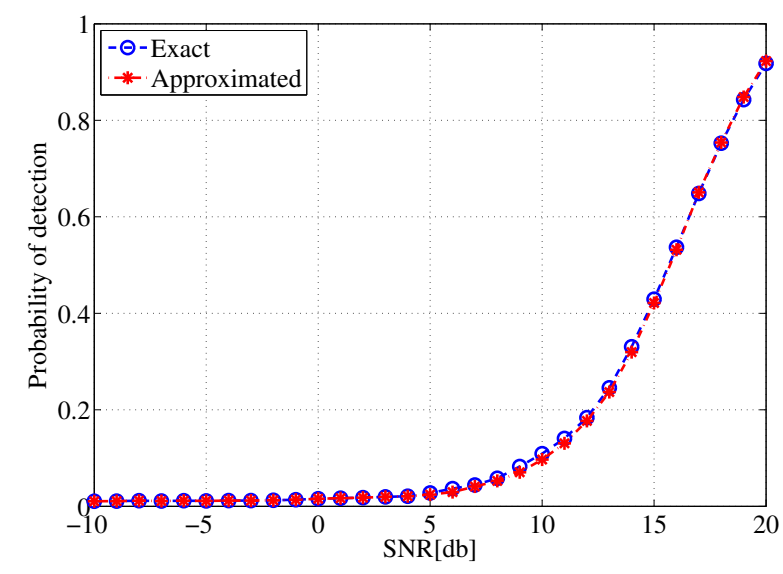

Fig. 1: Probability of detection of theoretical and approximated detector when $P_{F A}=0.01$ and $K=1$.

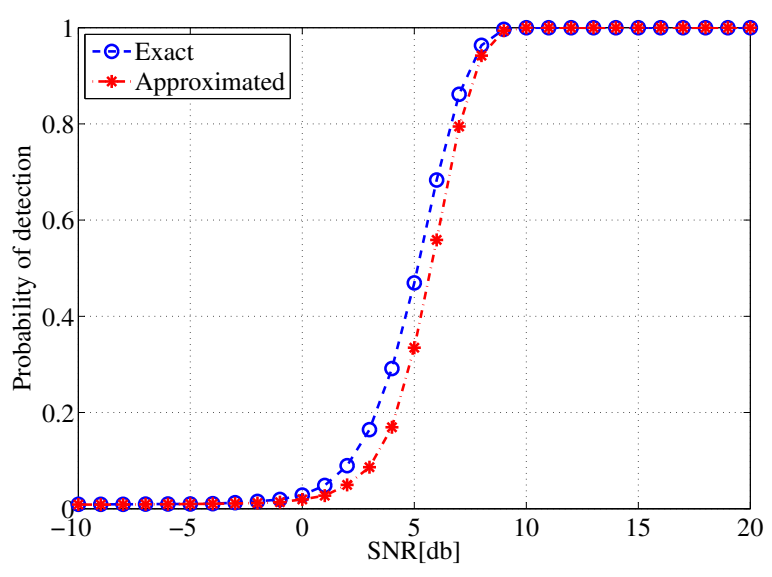

Fig. 2: Probability of detection of theoretical and approximated detector when $P_{F A}=0.01$ and $K=50$.

SNR(signal to noise ratio) is varied from the range of $(-10,20) \mathrm{dB}$ with the interval of $1 \mathrm{~dB}$. During the comparison between different detection methods, in each method $10^{4}$ channels are generated. Simultaneously the false alarm $P_{F A}$ is selected as $P_{F A}=0.01$, and the threshold $\gamma$ is determined by the false alarm $P_{F A}=\alpha$ as in (6).

Fig. 1 and Fig. 2 compares two detection methods. The curve named Exact is the detector corresponding to (12) with a higher computational complexity, while the one named Approximated is from the cost function (17), which requires relatively lower computations. As the number of samples $K$ increased, the performance of detection gets better in both detection methods. When SNR is low, the detection is poor since the noise effect is large. But good detection can be seen in a high SNR, since signal power is much greater than noise power. We can not find a significant difference be- 362tween two curves. It means that our proposed method 


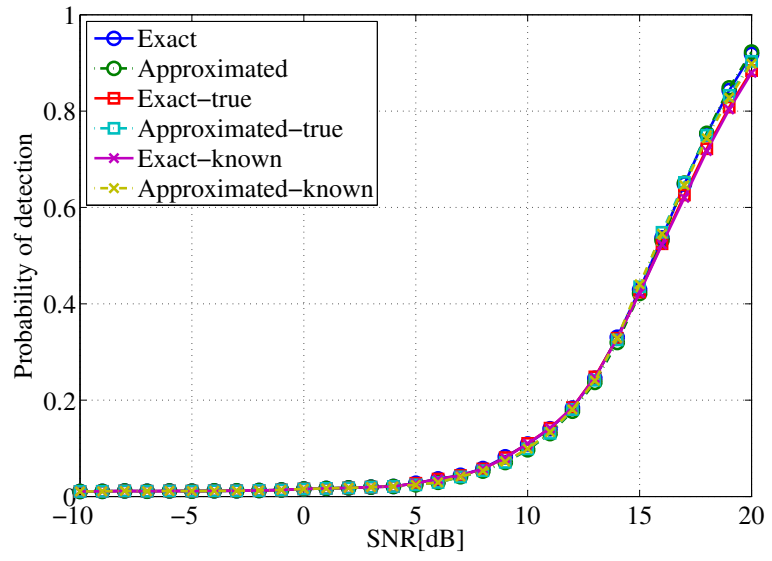

Fig. 3: Probability of detection with different $\sigma_{s}^{2}$ when $P_{F A}=0.01$ and $K=1$.

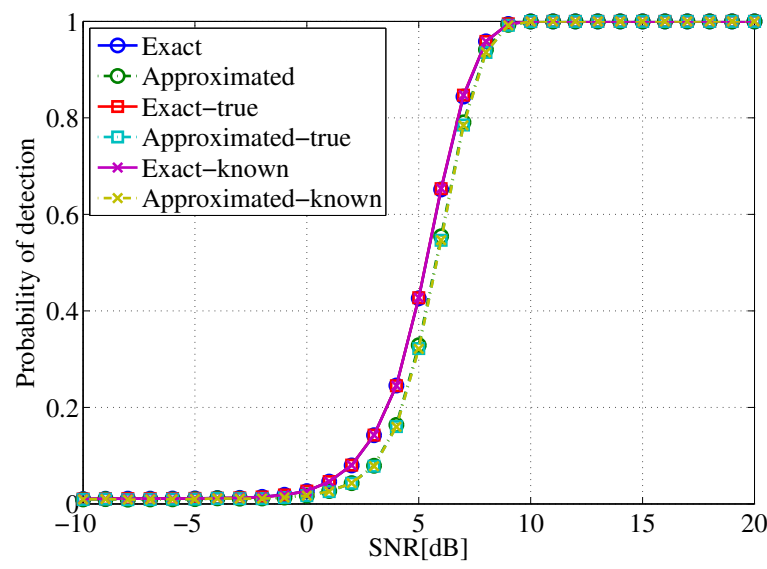

Fig. 4: Probability of detection with different $\sigma_{s}^{2}$ when $P_{F A}=0.01$ and $K=50$.

with reduced computation shows a good performance.

If we compare the $\sigma_{s}^{2}$ in our proposed method with the true $\sigma_{s}^{2}$ and the estimate $\hat{\sigma}_{s}^{2}$ in (19), the difference can be observed in Fig. 3 and Fig. 4. Since the difference between them is rather small, it shows that our proposed method works well in the detection of the modulation type of the transmitted symbols, even when we have finite samples.

\section{Conclusions}

The problem to detect modulation type of received signals over flat fading channels by using automatic modulation classification (AMC) is studied. First we design a method to determine the modulation type of received signals based on the Neyman-Pearson (NP) detector. Since the theoretical likelihood ratio function has large computations, to reduce the numerical com- plexity, a method by using probability density function (PDF) approximation is developed. To certify our proposed method has little effect by the uncertainty in noise variance, we also derive and compare different cases of whether the noise variance is known or not.

\section{References}

[1] A. Ghasemi and E. Sousa, "Spectrum sensing in cognitive radio networks: requirements, challenges and design trade-offs," IEEE Communications Magazine, vol. 46, pp. 32-39, April 2008.

[2] T. Yucek and H. Arslan, "A survey of spectrum sensing algorithms for cognitive radio applications," IEEE Communications Surveys Tutorials, vol. 11, no. 1, pp. 116-130, 2009.

[3] Y. Xiao and F. Hu, "Cognitive radio networks," Auerbach Publications, 2009

[4] S. Haykin, D. Thomson, and J. Reed, "Spectrum sensing for cognitive radio," Proceedings of the IEEE, vol. 97, pp. 849-877, May 2009.

[5] H.-C. Wu, M. Saquib, and Z. Yun, "Novel automatic modulation classification using cumulant features for communications via multipath channels," IEEE Transactions on Wireless Communications, vol. 7, pp. 3098-3105, August 2008.

[6] S. M. Kay, Fundamentals of Statistical Signal Processing, vol. 2. Prentice Hall, 1998.

[7] G. V. Moustakides, "Finite sample size optimality of GLR tests." Submitted to IEEE Transactions on Information Theory, Available at http://arxiv.org/pdf/0903.3795v2.

[8] J. Font-Segura and X. Wang, "GLRT-based spectrum sensing for cognitive radio with prior information," IEEE Transactions on Communications, vol. 58, pp. 2137-2146, July 2010. 